\title{
Real-Time Data Management for Network Information System
}

\author{
Dong Chun Lee \\ Dept. of Computer Science Howon Univ., South Korea \\ 727, Wolha-Ri, Impi, KunSan, ChonBuk, Korea \\ ldch@sunny.howon.ac.kr
}

\begin{abstract}
Network system, such as switching system, are operated in the restricted conditions that the suggested events must finish in the time-constraints. In this paper, we propose the data model which is suitable to the unstructured multi-dataset environment and the algorithm to clarify the structure of our model. The proposed model supports the fast data access for unstructured dataset and enables users to easily retrieve data needed at the execution.
\end{abstract}

\section{Introduction}

The complexity of data in the network system is not so serious that the current network environment can be represented as the structured dataset and can be used enough to retrieve information just like Data Lookup. This characteristic in the communication application doesn't have any trouble ensuring the time-constraint process of data [1. The recent network system has been through with various changes and the network environment should be connected with the plenty of services. The previous structured dataset saving and managing can come with limits of processing time and expansion. And with the increasing requirement of the communication common carrier for the software of network system that is compatible with open system, the definition and management method of communication data that have more complex and comprehensive semantic than the previous models are needed. In addition to it, the data throughput of the service systems has been sharply increasing in proportion to the data transmitting speed that is also getting faster result from the vitalized network services on the high-speed network. Consequently, faster data processing for the plenty of the data of complicated structure are imperative [2] [3]. Generally database system is used as a way of data management. However, data processing system based on the disk is excessively big size and has disappointing data processing performance. It is not suitable in the aspect of data processing speed and system adaptation required in the communication environment. Thus, every dataset to be operated is made resident in the main memory in order to offer very fast and uniform accessing time in the time-constraint system environment 
such as switching system and it is made to access with consistency and high speed [1].

This paper proposes the data model that can easily configuration the unstructured scheme. This model makes each data system have the unique Record Identifier (RID) in it, and has the complex relations and constraints that are required in the communication environment, represented consistently and shows the way of ensuring rapid processing performance through the navigation among the Record Identifiers (RID) without any physical reconstruction of the datasets at the request of multiple data.

\section{Related Work}

DREAM-S [1] has been used to manage call process and subscribers' data in the network system for the last years and it manages the information of the real world as a type of structured table. In the related data of routing process in switching center, dataset D-RT-RESOURCE-INF saves and manages the information about the root resource and D-SRT-RESOURCE-INF maintains the physical location information in the system that the sub-root belongs to. Meantime, each dataset is managed independently without any data model to clarify the correlations and constraints depending on designed semantic among datasets. The dataset, which has this type of construction, makes its structure and process simplicity. But it has a lot of restraints to solve the problems because it cannot read the semantic among the datasets due to the absence of the model. Unfortunately, the guarantee of the constraints makes the application systems more complex and it cause degrade of whole performance owing to the frequent access to the data processing system.

And also, when it is required to retrieve the information about the multiple datasets rather than to search a dataset as a simple lookup type, the Cursor is definitely needed as a tool that can locate the reconstructed dataset in the system, and support the consecutive access to the data [6. The Cursor is a kind of structure used to access orderly to the data that are correspondent to current query context. The reason the Cursor is needed in the data processing system is that database system deals with the group of data that is not restrained in the size of them, and the general programming language doesn't have any specific means to deal with data. Since the previous Cursor saves the result data from the data service separately in the main memory as much as the size of it in order to allow the access orderly to the application program, it produces the problem of wasting the saving space in the main memory and it needs additional time and algorithm to construct extra system for the access to the pertinent saving space. Currently the structured scheme based on database set presents various solutions, but the structured scheme, that can store other different datasets in it, is very inefficient and complex in processing. 


\section{Data Model of the Unstructured Dataset}

We describe the functional things provided by the system. That is, it describes more details about the composition of basic Dataset to operate a Cursor and the processes to recompose the related data through the Cursor. Dataset is the group of data with same quality or attribution. The data has inherently and means the data collection in the same type. Each data is uniqueness in the system, it was distinguish by the identifier with the physical location (that is, RID). The relations of the data are defined by regarding the related Identifier in the data as the attributions. For example, in the Figure 1, if there are any relations, Rc1-c2, between Dataset $\mathbf{C 1}$ and Dataset C2, then related two data, oc1 $\in \mathbf{C 1}$, oc2 $\in \mathbf{C 2}$, have each attribution as the identifier oc2, oc1. And REF, SET and Inverse are defined to clarify the relations and the multiplicity of the relations.

\begin{tabular}{|c|c|}
\hline $\begin{array}{c}\text { Create Dataset C1 (Attrel Rc1_c2 } \\
\text { REF C2) }\end{array}$ & Create Dataset C2(Rc2_c1 \\
SET inverse C1, Rc1_c2, Attrc2) \\
\hline
\end{tabular}

Fig. 1. Definition of the relationship in Dataset

In the above example, oc1 is unique relation with data in $\mathbf{C 2}$, on the other hand, oc2 can show the relation with oc1 as well as several data in $\mathbf{C 1}$ since the Rc2_c1 is declared by SET. Furthermore, Inverse means that it has always the both directions in the relations used Rc1_2 and Rc2_1 between $\mathbf{C 1}$ and C2, which $\mathbf{C 2}$ can be referred in $\mathbf{C 1}$ by using $\mathbf{R c 1} \mathbf{2}$ and $\mathbf{C 1}$ can be also referred by using Rc2_c1 in reverse, and it means the guarantee about Referential Integrity between the two data. The clarification of the relation is made when the data has its identifier. For example, getting oc2 in the related C2 data specifies Rc1-c2 in $\mathbf{C 1}$ data. The specification of oc1 and oc2 into Rc1_2 means that it's possible to access to oc2 data through oc2.Rc1_2, and, in reverse, it's also possible to access oc1 data through oc2.Rc2_c1. The previously defined relationship between Datasets are shown as the starting root with basic dataset when it is used in the query to retrieve the data from the system, the retrieval of the data was complete by using the identifier which the attribution has in the specified path.

Figure 2 shows the query about the Join of C1 and C2 with the application of the above. As it is seen in the query, the reference to the other data through Identifier Rc1_c2 or Rc2_c1's value means the Join, therefore the path operator ("->") is used to refer to other data through Identifier. Path operator is used when it designates the other related data, it can be iterative joined with another data through the identifier in the designated data. 


Retrieve Attrel, Rc1_c2- $\_$Attrc2 from C1
Retrieve Rc2_c1- > Attrc1, Attrc2 from C2

Fig. 2. Definition of the relationship in Dataset

In case that the Join is needed, it can be accessed to other Dataset, C2 or C1 directly by using Rc1_c2 or Rc2_c1of C2 from C1, and it's possible to access without creating temporary Dataset so that it makes the system search faster than the previous methods [4] [5].

Furthermore, in the communication environment, operating to retrieve the minor data which is identical to the condition is more often required than the operation to retrieve the entire Dataset after the Join, which often reconfiguration of the Dataset have many effects on the data processing performance in Joining [1]. And it is mainly described the navigation algorithm necessary for Join between related data with the identifier to express the relationship in the data model and the structure of the algorithm.

\section{Navigation-Based Dataset Cursor in Implementation}

Cursor is the structure to be used for the sequential access operation in the data of successful Query. While the previous cursor provides consecutive access to the recomposed data, the proposed method using the RID is described more flexible and effective cursor by direct access to the data through the navigation with no physical reconfiguration of the data.

\subsection{Structure of the Cursor}

The Cursor can be described with following four elements. At first, D is the dataset that has the information to be retrieved, and $\mathrm{A}$ is the group of data RID that are objects of search in D. The group of RID is distinguished by the path, and refers to the group of data that becomes the object of operation at the same time as the operation. $\mathrm{C}$ is the conditions to be met in order to return to the result, and consists of attribute, operator, and value. $\mathrm{S}$ indicates the attribution information retrieved when it meets the condition; Cursor $\mathbf{C s r}=(\mathbf{D}, \mathbf{A}, \mathbf{C}, \mathbf{S})$ where D is Set of dataset, A is Set of data RID (=Alias) of the searching object in $\mathrm{D}, \mathrm{C}$ is Set of condition expression and $\mathrm{S}$ is Set of result attribute.

For instance, the Cursor c' $\in$ Csr that consists of "retrieve Rcl c2->Attrrc 2 from $\mathrm{C} 1$ where Attre1=10" in the definition of figure 2 can be expressed $\left(\{\mathrm{C} 1, \mathrm{C} 2\},\{\{\right.$ oc1 $\},\{$ oc1.Pc1_c 2$\}\},\{$ Attrc1=10 $\left.\},\left\{\mathrm{Rc} 1 \_\mathrm{c} 2->\operatorname{Attrc} 2\right\}\right)$. The oc1 is the RID referring to the data in the $\mathrm{C} 1$, and oc1.Rc1_c2 is the RID referring to $\mathrm{C} 2$ related to oc1. Therefore, oc1.Rc1_c2->Attrc2 refers to specific character of 
C2 data. To process the query, the Cursor is composed through the analyzing process of the query, and the result is retrieved through the navigator. The tree that uses the basic dataset as a root is built up when referring to other dataset through the relations like REF and SET from the basic dataset in the query, namely when the path is specified.

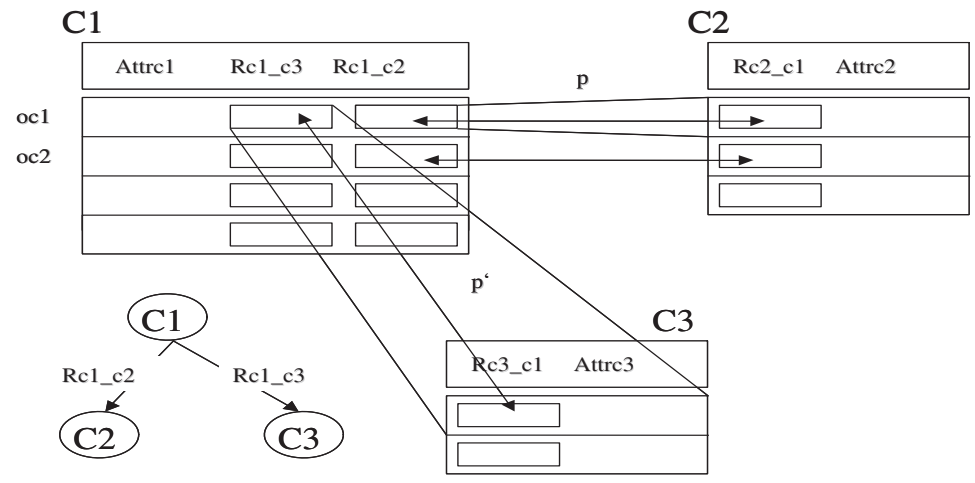

Fig. 3. Hierarchical structure of the path

Figure 3 shows that Rc1_c2 refers to $\mathrm{C} 2$ in $\mathrm{C} 1$ and $\mathrm{Rc} 1 \_\mathrm{c} 3$ can be the path to C3. Thus, the attribution in $\mathrm{C} 2$ using $\mathrm{C} 1$ as a path can be referred through the path expression that uses the Rc1_c2, the $\mathrm{C} 1$ character, and the character in C3, through the Rc1_c3. Moreover, the condition expression can specify by using the path expression. Here reference means Rc1_c2 and Cc1_C3 in C2 has the RID for the specific data in each C2and C3. Representing relations and retrieving attributions between datasets $\mathrm{C} 1, \mathrm{C} 2$ and $\mathrm{C} 3$ by accessing directly to the data in $\mathrm{C} 2$, and $\mathrm{C} 3$ can be possible by solely systemized representation, path expression.

\subsection{Retrieval of Alias in Query}

To connect and operate different and independent dataset through RID, the path to distinguish its dataset must be identified and be correspondent to the effective RID within its RID group. This is because the object data of C2 and C3 have to be changed together according to the object of operating in $\mathrm{C} 1$ changed from oc1 to oc2. This is the course of retrieving the RID of actual $\mathrm{C} 2$ corresponding to the attribution value of the specific data in the path p of $\mathrm{C} 1$ (Pc1 (Pc2_c2)-> C2). The change of Rc1_c2 into Rc1_c2' means the operating object of $\mathrm{C} 2$ has to be changed to Pc1 (Rc1_c2'). For this case, each path existing in the datasets must be analyzed and RID group correspondent to them should be produced. 
This is called the Alias and it is defined as follows; Alias $\mathbf{A}=(\mathbf{D}, \mathbf{P}, \mathbf{F})$ where $\mathrm{D}$ is Dataset which refers to the current dataset, $\mathrm{P}$ is The path that distinguish the current $\mathrm{D}$ with previous Alias and attribution and $\mathrm{F}$ is REF/SET attribution.

Alias be distinguished by the paths referring to datasets. That is, the fact that $\mathrm{C} 2$ has the one path passing through only Rc1_c2 from the all the data in C1 shows that there is only one Alias.

Suppose the situation that several aliases can exist in one dataset. For example of C3, both RID by Rc1_c3 and RID referred by Rc3_c3 refer to the data in C1, but it must be classified by the context referring to $\mathrm{C} 3$. As a result of it, it is defined to only be distinguished by the previous dataset referring to itself in the path in order to distinguish the several RID in the dataset, its attribute, and all the three elements (dataset, character and path) composed by the compounded path. But, the basic dataset is defined to have the basic default path. For example, $\mathrm{C} 1$ has only the default path, C3 has the path of C1-C3, (C1, Rc1_c3, 0) (Here, this is the default path ID of $\mathrm{C} 1$ which is the basic dataset of 0 ). If $\mathrm{C} 1$ has self-reference structure, it will have additional (C1, Rc1_c1 and 0) except for the default path. And if it has the relations with $\mathrm{C} 3$ through (C1, Rc1_c1 and 0), C3 will have (C1,Rc1_3) and (C1, Rc1_c1, 0) additionally. This path is used to retrieve the Alias (= RID of dataset) which is the object correspondent to the current context in the course of navigating the classified dataset through the RID.

The Figure 4 is more specified figure 4 based on Alias. The basic dataset $\mathrm{C} 1$ (=BASE_dset) is initialized to have the basic Alias al(=BASE_alias). Alias a2 of dataset $\mathrm{C} 2$ is produced by BASE_alias, al and Rc1_c2.When Rc1_c2', the relation from $\mathrm{C} 1$ to $\mathrm{C} 2$, has already been defined, this path produced new alias a3(Base_alias, al and Rc1-c2').

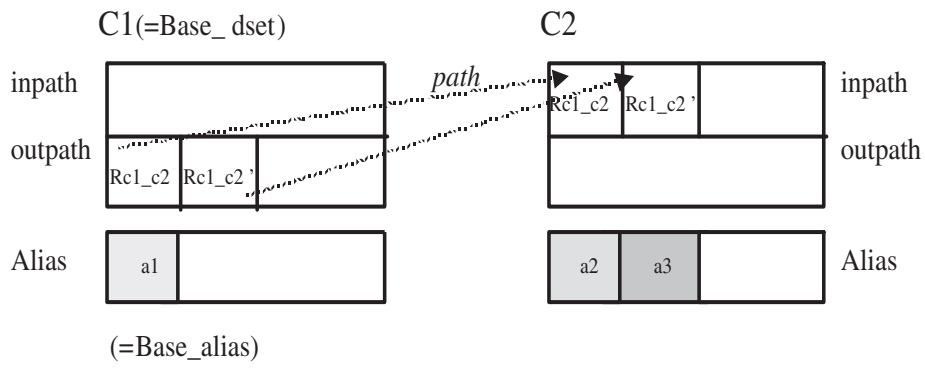

Fig. 4. Retrieval of the Alias 
The following algorithm is the one to produce Alias by analyzing the path expression when basic dataset and the path expression are given. This path expression has the form of " $\mathrm{s}->\mathrm{n}->$..." and $\mathrm{s}$ and $\mathrm{n}$ are the names of attributions that are the types of REF or SET referring to each dataset.

Algorithm Makes Cursor (name: dataset name, p: path expression) Dset: dataset information

Alias: working Alias begin

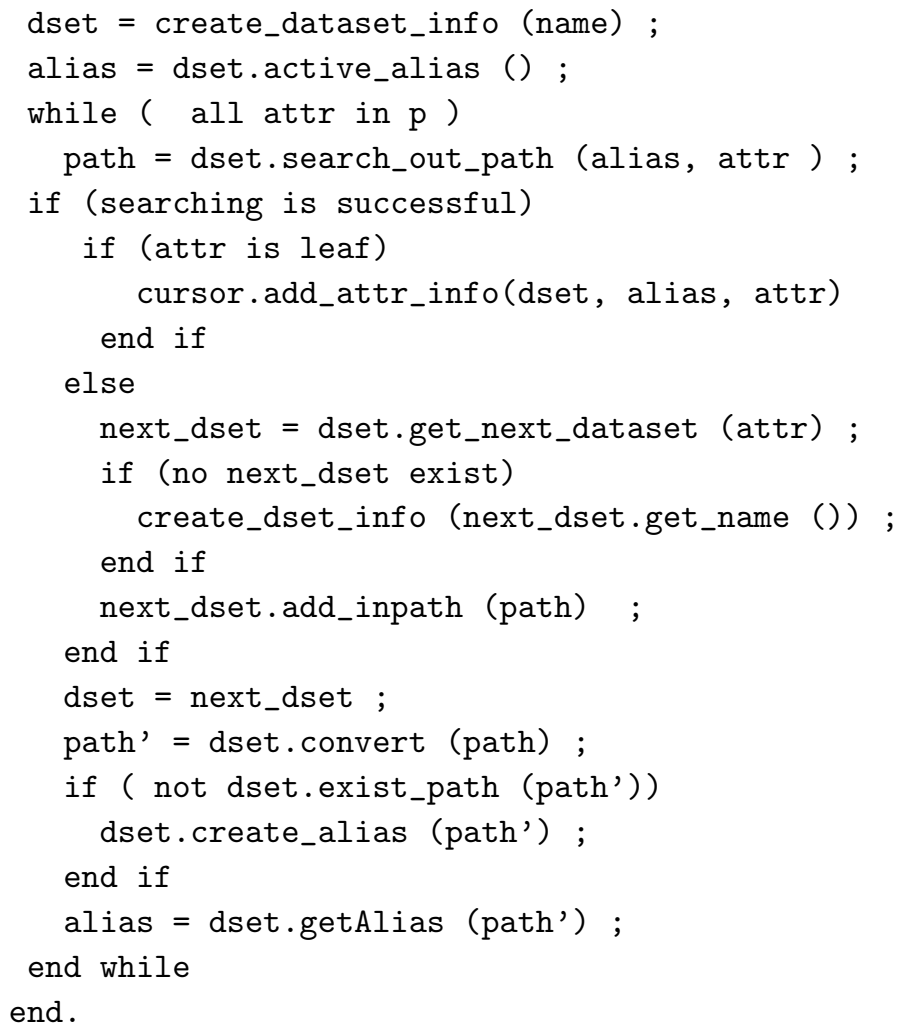

This algorithm is noticed that new alias is produced when the path to the present attribution of present dataset doesn't exist.

\subsection{Navigation Using the Alias}

The composed Alias is needed to navigate the dataset. The way of navigation is made up be the evaluation of the condition expression. For this case, the Alias including the attribution stated in the condition expression evaluates the referred data and expression. If the evaluation of all the expression is successful, all the data referred by alias become the object of query result. If not, next data RID is replaced by alias and repeat the evaluation of the expression. The figure 6 
shows such a process course, which it is 2 aliases in $\mathrm{C} 2$. If the alias a3 is defined that it has a relations with $\mathrm{C} 1$ and SET, and if the evaluation of the condition expression about the data referred by current a3 (that is, RID is oc3) is not true, the a3 evaluates the expression replaced by possible next data RID, oc4. After the evaluation of the expression, the result is not true again, next RID is retrieved to replace a3. By the time it is not possible to retrieve the next RID, it is required (touch_coursor in algorithm) to replace alias a1 in C1 which is more upper path than a3. If a1 which is either REF or SET doesn't have any RID to replace, it is necessary to replace a1 itself. Namely, the next data except for oc1 is newly referred by a1 in C1. According to it, the lower alias (a2 and a3) than a1 are initialized by a1 and then begins to estimate the former expression.

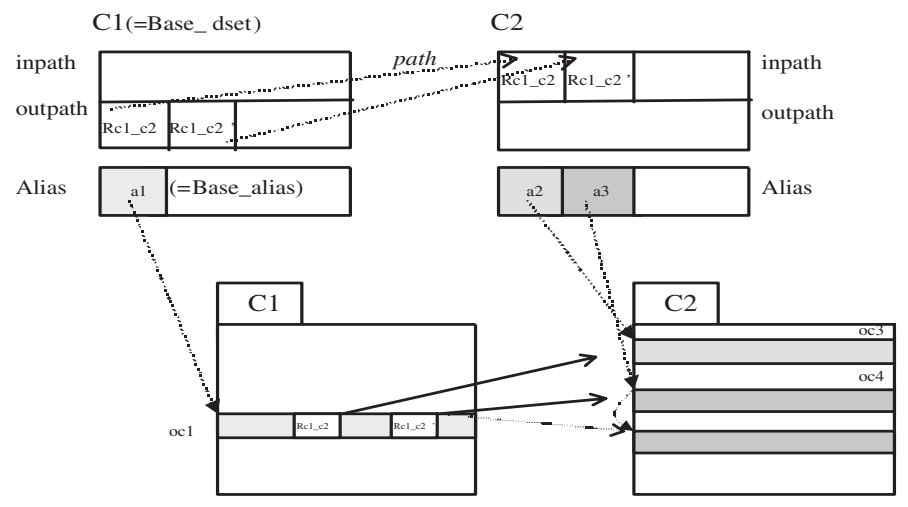

Fig. 5. Navigation of Dataset

By repeating this process, the results having evaluated all the data in C1 returned to the user. The following algorithm shows the course of evaluating the condition expression given on the basis of dataset and retrieving alias.

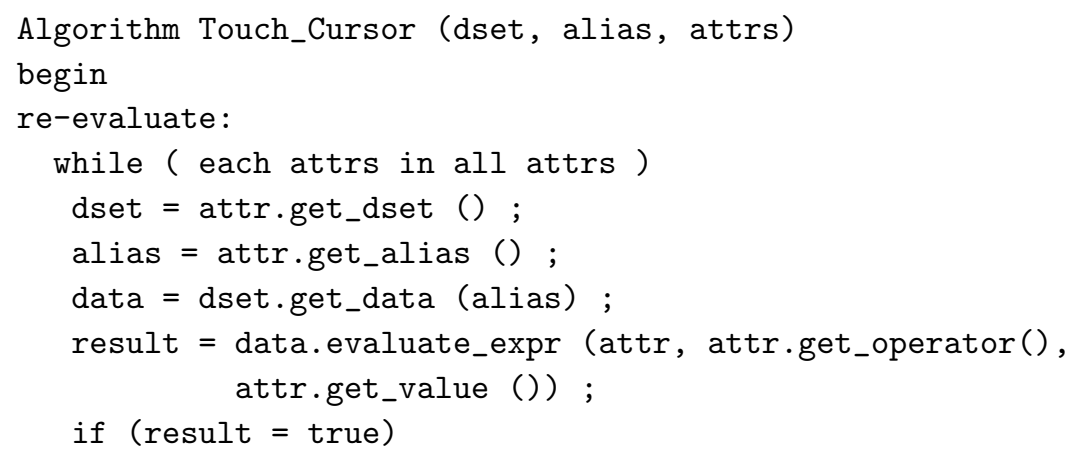




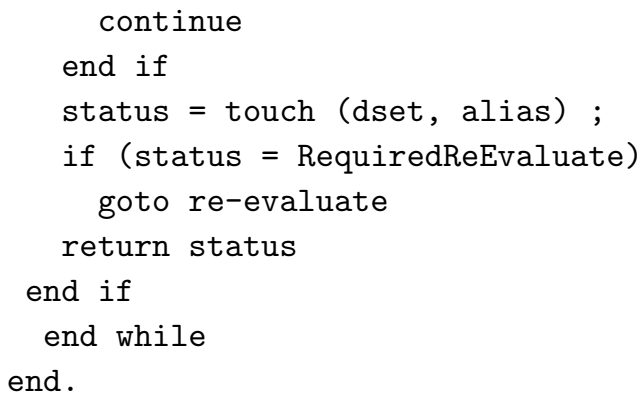

The basic way to replace Alias is to require replacement of upper alias on the path when the alias itself cannot meet the condition evaluation. And also, the following algorithm shows the procedure to require the replacement of upper alias.

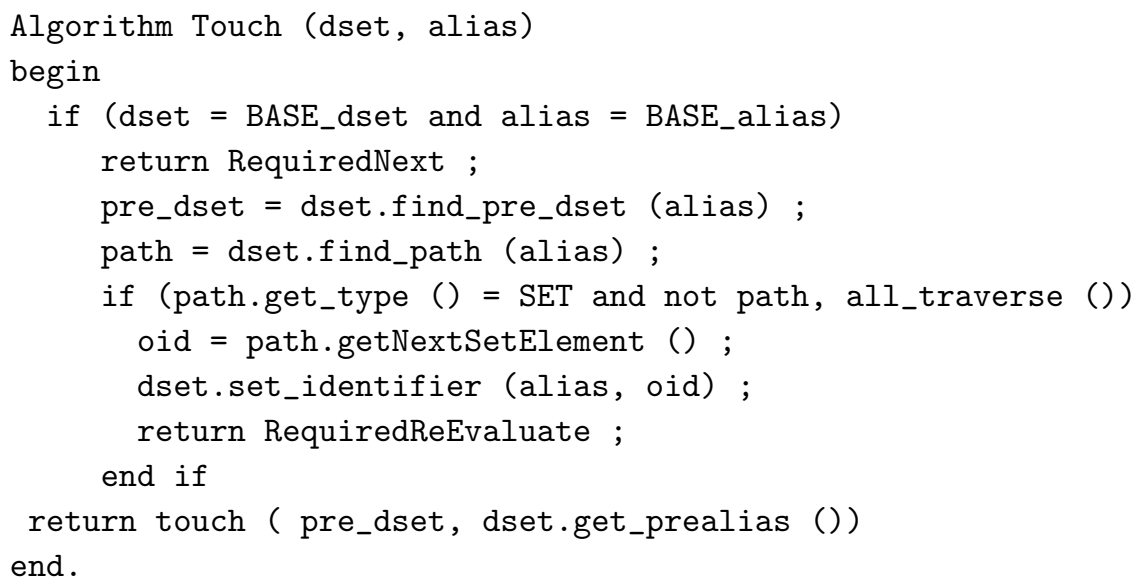

In above algorithm, the RequeiredReEvaluate means that the condition evaluation given in query has to be reevaluated after being replaced by lower alias (a2 or a3) without any change of basic alias (For example, a1 in C1).

\subsection{Retrieval Result}

When all the condition evaluation are evaluated successfully, the data referred by current alias in each dataset have to be returned. If the query clarifies the specific character of data, only that specified attributions have to be retrieved and returned. For this case, the Cursor has the list of the result attributions and the result attributions are defined as following three elements; Result attributions $\mathbf{R S}=(\mathbf{A}, \mathbf{P}, \mathbf{S})$ where $\mathrm{A}$ is Dataset and alias where each attribution belongs, $\mathrm{P}$ is The location value of result value at the beginning of the data referred as alias and $\mathrm{S}$ is Actual size of the result value. 
For instance, actual value states that attr3 is as big as size 2 and it has the same offset location as ofs1 of data (oc3) referred by alias a2 in dataset C2. Therefore, attr 3 can be presented as (a2, ofs 1 and size1) $\in \mathrm{RS}$, and attr 4 can be also described as (a3, ofs2 and size2) $\in$ RS because it refers to the character in the data $0 \mathrm{c} 4$, referred by alias a3 in the same dataset. The composition of the result value to be returned without considering multiple dataset is possible by cursor's retrieving continually the value as much in the location of data offset which specified alias refers to in the list of result characters.

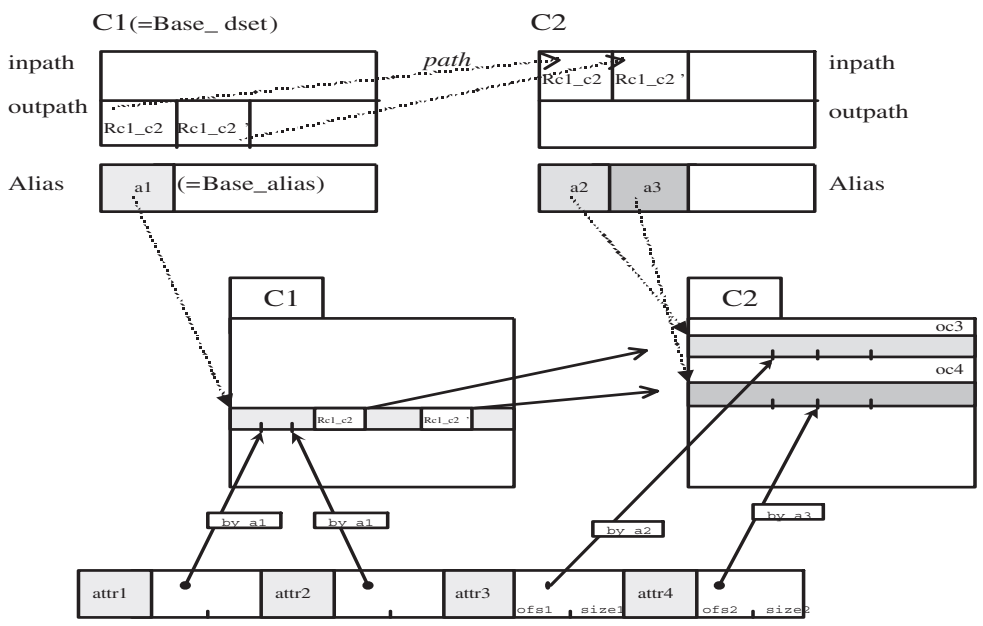

Fig. 6. Data retrieval

In the algorithm, attrs means the list of result attribution and results are the buffers to save the actual value of the attributions. When they are added to the result buffer, each attribute has the type and size information additionally.

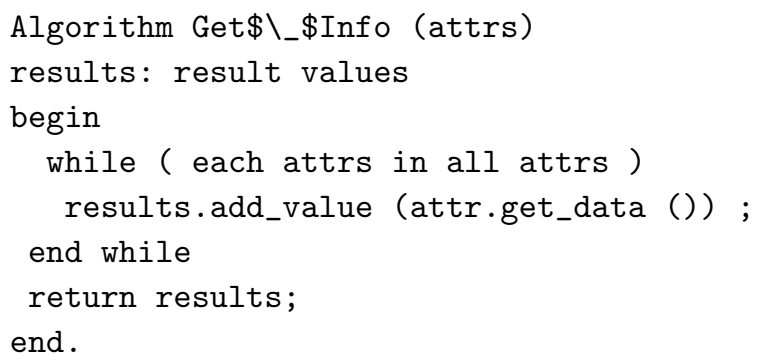

As shown above, the data retrieving method through Alias navigation makes it possible to retrieve the results without any physical recomposition compared to 
previous methods [1] [4] [5] that recompose all the dataset in the query physically. This function offers rapider performance and more uniform access performance in retrieving many results.

\section{Conclusion}

When joining multiple dataset, previous methods are using the way of recomposing new dataset or index by copying a lot of memory though it is the database in the memory. As a result, it cannot avoid the bottleneck problem [7] of the memory which has to be minimized in terms of current hardware structure and this may consume much preparing time depending on the size and the state of the dataset 8 .

We propose the Join method which minimize the physical repeating data service between memories. This method makes it possible to get the results, tracing the addresses of memories without any physical memory copy according to the relations at the request of retrieving. More specifically, we are proposed structured access system and interface through the data model that is including path expression in term of the dataset that has the unstructured and complex structure.

As a result, the proposed method makes the unstructured scheme represented easily in the communication environment and enables us to access to data easily, and meets the time-constraints of event which can be made up at the time of dataset Join and has the effect in reducing the complexity of the application program.

Acknowledgments. This work is supported by Howon University Fund 2003.

\section{References}

1. Y.I. Yoon, et. al., "Scalable Distributed Real-time Database Management for Switching System," Int'l Switching Symposium (ISS'97), Toronto, Canada, pp. 539545, September 1997.

2. M. Stonebreaker and P. Brown, "Object-Relational DBMSs: Tracking the Next Great Wave," Morgan Kaufmann Publishers, pp. 320, 1998

3. W. Wolfe, J. Prichard, L. Dipippo, and J. Black, "Real-Time Database Systems: The RTSORAC Real-Time Object-Oriented Database Prototype," Kluwer Academic Publishers, pp. 279-303, 1997.

4. Polyhedra Plc., "Bloor Research Overview of Polyhedra," White Paper, http://www.polyhedra.com

5. Raima Co., "Raima Database Manager++ In Real-time and Embedded Systems," White Paper, http://www.mbrane.com/rdm.html

6. C. T. Yu and W. Meng, "Principles of Database Query Processing for Advanced Applications," Morgan Kaufmann Publishers, pp. 512, 1997. 
7. Peter Boncz, Stefan Manegold, Martin Kersten, "Data Architecture Optimized for the New Bottleneck: Memory Access," Proc. of VLDB'99, 1999.

8. H. Garcia-Molina and K. Salem, "Main Memory Database Systems: An Overview," IEEE Transaction on Knowledge and Data Engineering, Vol. 4, No. 6, pp. 509-516, Dec. 1992. 